\title{
LIMIT THEOREMS FOR DIVISOR DISTRIBUTIONS
}

\author{
MICHAEL D. VOSE
}

\begin{abstract}
For a positive integer $N$, let $X_{N}$ be a random variable uniformly distributed over the set $\{\log d: d \mid N\}$. Let $F_{N}$ be the normalized (to have expectation zero and variance one) distribution function for $X_{N}$. Necessary and sufficient conditions for the convergence of a sequence $F_{N_{i}}$ of distributions are given. The possible limit distributions are investigated, and the case where the limit distribution is normal is considered in detail.
\end{abstract}

1. Introduction. Let the positive integer $N$ have prime factorization $N=p_{1}^{\alpha_{1}} \cdots p_{k}^{\alpha_{k}}$. Define $\mu_{n}(N)$, for positive integer $n$, by

$$
\mu_{n}=\left(12^{-1} \sum_{1 \leqslant j \leqslant h}\left(\left(\alpha_{j}+1\right)^{n}-1\right)\left(\log p_{j}\right)^{n}\right)^{1 / n} .
$$

The divisor distribution of $N$ refers to the function

$$
F_{N}(x)=\tau^{-1} \sum_{d \mid N}^{\prime} 1
$$

where $\tau$ is the number of divisors of $N$, and the sum is restricted to those divisors satisfying $\log (d / \sqrt{N}) \leqslant x \mu_{2}$.

In this paper, we determine when a sequence of divisor distributions tends to a limit, and investigate the limit distributions that arise. Erdös and Nicolas [2] had previously shown the divisor distribution of $N_{j}=\prod_{p<j} p$ (we reserve the letters $p$ and $q$ for primes) to be asymptotically normal as $j \rightarrow \infty$. With regard to the normal distribution we prove

THEOREM 2. The normal distribution

$$
\Phi(x)=(2 \pi)^{-1 / 2} \int_{-\infty}^{x} \exp \left(-\frac{1}{2} t^{2}\right) d t
$$

is the only infinitely divisible distribution that can arise as the limit of a sequence $F_{N}$, of divisor distributions. A necessary and sufficient condition for convergence is that

$$
\lim _{j \rightarrow \infty}\left(\mu_{2}\left(N_{j}\right)\right)^{-1} \mu_{\infty}\left(N_{j}\right)=0
$$

Moreover,

$$
\sup _{w}\left|F_{N}(w)-\Phi(w)\right| \ll \frac{\mu_{\infty}}{\mu_{2}}
$$

Received by the editors November 11, 1984 and, in revised form, February 1, 1985.

1980 Mathematics Subject Classification. Primary $10 \mathrm{~L} 10$. 
and

$$
\left|\frac{1-F_{N}(x)}{1-\Phi(x)}-1\right| \ll\left(x+\frac{1}{x}\right) \frac{\mu_{4}}{\mu_{2}}
$$

for $x \leqslant \mu_{2} \mu_{4}^{-1}$.

Here $\mu_{\infty}(N)$ means $\lim _{n \rightarrow \infty} \mu_{n}(N)$.

We define the (Fourier) transform of a distribution $F$ as

$$
\hat{F}(t)=\int_{R} e^{2 \pi i t x} d F(x) .
$$

If $\hat{F}(t)$ is the restriction to $R$ of an entire function, we say that $\hat{F}$ is entire. In the general case we have

THEOREM 1. A necessary and sufficient condition for a sequence $F_{N_{1}}$ of divisor distributions to converge to a distribution $F$ is that for each $n$ the limits $a_{n}=$ $\lim _{j \rightarrow \infty} \mu_{2 n}\left(N_{j}\right)\left(\mu_{2}\left(N_{j}\right)\right)^{-1}$ exist. In this case $\hat{F}$ is entire and is represented in the disk $|z|<1 / 4$ by

$$
\hat{F}(z)=\exp \left(-\sum_{n=1}^{\infty}(n(2 n) !)^{-1} 6 B_{n}\left(2 \pi a_{n} z\right)^{2 n}\right)
$$

where

$$
B_{n}=4 n \int_{0}^{\infty}\left(e^{2 \pi t}-1\right)^{-1} t^{2 n-1} d t
$$

are the Bernoulli numbers.

We say that a sequence $F_{j}$ of distributions converges to $F$, if $F_{j}( \pm \infty) \rightarrow F( \pm \infty)$, and $F_{j}(x) \rightarrow F(x)$ at all continuity points $x$ of $F$.

A reasonable characterization of the possible limit distributions seems difficult. We do however have the following "factoring theorem":

Theorem 3. Suppose the sequence $F_{N_{i}}$ of divisor distributions converges to $F$. If $F$ is not a finite point mass distribution then, for some $\phi \in[0, \pi / 2)$,

$$
F(x)=G(x \sec \phi) * H(x \csc \phi) .
$$

The convolution factor $G$ is a normal, uniform, or singular distribution. $H$ is the limit of a sequence of divisor distributions when $\phi>0$, and otherwise is to be interpreted as point mass at 0 . Moreover, if $\liminf _{j \rightarrow \infty} \omega\left(N_{j}\right)=K<\infty$, then $F$ may be written as a convolution product involving no more than $K$ uniform or arithmetic distributions.

Here $\omega(N)$ is the number of distinct prime divisors of $N$. A finite point mass distribution is a finite convolution of arithmetic distributions. An arithmetic distribution is a probability distribution with zero expectation, and, a step function, whose finitely many jump discontinuities are of equal height and occur along an arithmetic progression. A uniform distribution has density $(12)^{-1 / 2} \chi_{[-\sqrt{3}, \sqrt{3}]}$ (where $\chi_{I}$ is the indicator of the interval $\left.I\right)$, and a singular distribution is continuous with zero derivative almost everywhere. 


\section{Necessary and sufficient conditions.}

LEMMA 1. The transform $\hat{F}_{N}$ of the divisor distribution of $N$ is an entire function which is represented in the disk $|z|<\mu_{2} \mu_{\infty}^{-1}$ by

$$
\hat{F}_{N}(z)=\exp \left\{-\sum_{n=1}^{\infty}(n(2 n) !)^{-1} 6 B_{n}\left(\mu_{2 n} \mu_{2}^{-1} 2 \pi z\right)^{2 n}\right\} .
$$

Proof. $\hat{F}_{N}$ is entire since $d F_{N}$ is compactly supported. For notational convenience, let $u=\pi \mu_{2}^{-1} t$. The transform of $F_{n}$ is

$$
F(t)=\prod_{j=1}^{k}\left(\left(\alpha_{j}+1\right) \sin \left(u \log p_{j}\right)\right)^{-1} \sin \left(u\left(\alpha_{j}+1\right) \log p_{j}\right) .
$$

Taking the logarithm of (2.1) yields

$$
\hat{F}(t)=\exp \left\{\sum_{j=1}^{k} \int_{u \log p_{j}}^{u\left(\alpha_{j}+1\right) \log p_{j}} \cot x-x^{-1} d x\right\} .
$$

Substituting the power series for $\cot x-x^{-1}$ in (2.2), integrating term by term, and interchanging the order of summation completes the proof.

Corollary 1. If $|y|<\lambda<1 / 4$, then $\hat{F}_{N}(x+i y) \ll_{\lambda} 1$. Also, $\hat{F}$ has a zero at $\mu_{2} \mu_{\infty}^{-1}$.

Proof. (2.1) shows that $\hat{F}_{N}\left(\mu_{2} \mu_{\infty}^{-1}\right)=0$. If $m$ is a positive integer, $m \leqslant 2 n$, then

$$
\mu_{2 n} \mu_{m}^{-1} \leqslant\left(1-2^{-m}\right)^{-1 / m} 12^{1 / m-1 / 2 n} \text {. }
$$

Since $\hat{F}_{N}(x+i y) \ll \hat{F}_{N}(i y)$, using inequality (2.3) in Lemma 1 completes the proof.

Proof of Theorem 1. By Corollary 1 , the collection $\mathscr{F}=\left\{\hat{F}_{N j}\right\}_{1}^{\infty}$ of analytic functions is uniformly bounded on compact subsets of $G_{\lambda}=\{x+i y \in \mathbf{C}:|y|<\lambda$ $<1 / 4\}$. Therefore, Montel's theorem [1] implies that any sequence of functions from $\mathscr{F}$ has a subsequence which converges uniformly on compact subsets of $G_{\lambda}$. This, together with the representation of $\hat{F}_{N}$ provided by Lemma 1 , implies that the sequence $\hat{F}_{N_{j}}$ converges to a function $H$ if and only if the limits $a_{n}$ exist, and in such case,

$$
H(z)=\exp \left\{-\sum_{n=1}^{\infty}(n(2 n) !)^{-1} 6 B_{n}\left(a_{n} 2 \pi z\right)^{2 n}\right\}
$$

in the disk $|z|<1 / 4$. It follows from the continuity theorem for Fourier-Stieltjes transforms that the convergence of $\hat{F}_{N_{j}}$ to such a function $H$ is equivalent to the convergence of $F_{N_{j}}$ to some distribution $F$, and in such case, $\hat{F}=H$. It remains to show that $\hat{F}$ is entire.

The inequality (for $x \geqslant 0$ )

$$
1-F(x) \leqslant F(-x) \leqslant \exp \left\{-x^{2} / 6\right\}
$$

implies that the sequence of entire functions

$$
H_{L}(z)=\int_{-L}^{L} e^{2 \pi i z x} d F(x)
$$


is uniformly Cauchy on compact subsets of $\mathbf{C}$. It therefore suffices to prove (2.4). Note that for positive $\lambda$ and positive $x$,

$$
1-F_{N}(x) \leqslant F_{N}(-x) \leqslant \tau(N)^{-1} \sum_{d \mid N} \exp \left\{-\lambda x+\lambda \mu_{2}^{-1} \log \frac{\sqrt{N}}{d}\right\} .
$$

With $\lambda_{j}=\lambda \mu_{2}^{-1} \log p_{j}$, the right-hand side of (2.5) is equal to

$$
e^{-\lambda x} \prod_{j=1}^{k}\left(\exp \left\{\frac{1}{2} \alpha_{j} \lambda_{j}\right\}\left(\alpha_{j}+1\right)^{-1} \sum_{n=0}^{\alpha_{j}} \exp \left\{-n \lambda_{j}\right\}\right) \text {. }
$$

Using the convexity of $e^{x}$ and the inequality $\cosh (x) \leqslant \exp \left\{x^{2} / 2\right\}$, we see that (2.6) is not greater than $\exp \left\{-\lambda x+\lambda^{2} / 2\right\}$. Choosing $\lambda=3^{-1} x$ finishes the proof.

Note that our method of proving Theorem 1 (via Montel's Theorem) shows that any sequence of $F_{N_{j}}\left(\right.$ or $\left.\hat{F}_{N_{j}}\right)$ has a subsequence which converges to some $F$ (respectively $\hat{F}$ ).

Proof of TheOREM 2. Suppose $F_{N_{j}}$ converges to $\Phi$. By Theorem 1, we have

$$
\hat{\Phi}(t)=\exp \left\{-\sum_{n=1}^{\infty}(n(2 n) !)^{-1} 6 B_{n}\left(a_{n} 2 \pi t\right)^{2 n}\right\} .
$$

On the other hand, $\hat{\Phi}(t)=\exp \left\{-2 \pi^{2} t^{2}\right\}$. It follows that $a_{j}=0$ for $j>1$. Conversely, if $a_{j}=0$ for $j>1$, then the sequence $F_{N_{j}}$ converges to some distribution $F$, where $\hat{F}(t)=\exp \left\{-2 \pi^{2} t^{2}\right\}$. Hence $F=\Phi$. It follows that $\mu_{\infty} \mu_{2}^{-1} \rightarrow 0$ is necessary and sufficient since

$$
\mu_{\infty} \mu_{2}^{-1} \ll \mu_{2 j} \mu_{2}^{-1} \ll\left(\mu_{\infty} \mu_{2}^{-1}\right)^{1 / 4}
$$

If $F_{N_{j}}$ does not converge to $\Phi$, then there is some compact interval of $\mathbf{R}$ containing infinitely many of the points $t_{j}=\mu_{2}\left(N_{j}\right)\left(\mu_{\infty}\left(N_{j}\right)\right)^{-1}$. Let $t^{*}$ be a limit point. Each $t_{j}$ is a zero of $\hat{F}_{N_{j}}$ by Corollary 1 , so if $F_{N_{j}}$ were to converge to $F$, then $F\left(t^{*}\right)=0$. This precludes the possibility that $F$ is infinitely divisible, since such distributions have positive transforms.

The first inequality of Theorem 2 is a straightforward application of the following result, referred to as the Berry-Eseen inequality. Let $F$ and $G$ be probability distributions, and suppose $G$ has density $g$. Then for all $T>0$

$$
\sup _{x}|F(x)-G(x)| \ll T^{-1}\|g\|_{\infty}+\int_{-T}^{T}|t|^{-1}|\hat{F}(t)-\hat{G}(t)| d t
$$

(Feller [3]).

To prove the second inequality of Theorem 2, define the measures $d V$ and $d G$ by

$$
d V(x)=e^{-A+y x} d F_{N}(x), \quad d G(x)=(2 \pi)^{-1 / 2} e^{-(x-y)^{2} / 2} d x .
$$

Let $R(x)=\exp \left(x^{2} / 2\right)(1-\Phi(x))$. Then

$$
\frac{1-F_{N}(y)}{1-\Phi(y)}=e^{y^{2} / 2} R(y)^{-1}\left(I+e^{A-y^{2}} R(y)\right),
$$

where

$$
I=e^{A} \int_{y}^{\infty} e^{-y x} d(V(x)-G(x))
$$


Now define $A-y^{2} / 2$ to be the function

$$
H(y)=-\sum_{n=2}^{\infty} 6 B_{n}(n(2 n) !)^{-1}\left(\mu_{2 n} \mu_{2}^{-1} i y\right)^{2 n} .
$$

Assuming that $|V(x)-G(x)| \leqslant \Delta$ and $y>0,(2.7)$ becomes

$$
\frac{1-F_{N}(y)}{1-\Phi(y)}=e^{H(y)}+O\left(\Delta R(y)^{-1} e^{H(y)}\right) \text {. }
$$

It is well known that $R(y)^{-1} \leqslant \sqrt{2 \pi}\left(y+y^{-1}\right)$ (see for example Mitrinovic [4]), so the proof is completed by establishing, for $0<y \leqslant \mu_{2} / \mu_{4}$, the inequalities

$$
|H(y)| \ll y^{4}\left(\mu_{4} / \mu_{2}\right)^{4},
$$

and

$$
\Delta \ll \mu_{4} / \mu_{2} .
$$

$H(m)$ is the sum of an alternating decreasing sequence, hence (2.9). The Berry-Eseen inequality, with $F=V$ and $G=G$, yields (2.10).

\section{The factoring theorem.}

LEMMA 3. If $M$ and $N$ are relatively prime positive integers, then

$$
\hat{F}_{M N}(t)=\hat{F}_{M}(t \cos \phi) \hat{F}_{N}(t \sin \phi)
$$

where

$$
\cos \phi=\frac{\mu_{2}(M)}{\sqrt{\left(\mu_{2}(M)\right)^{2}+\left(\mu_{2}(N)\right)^{2}}} .
$$

Proof. The functions $\left(\mu_{n}(N)\right)^{n}$ are additive. Therefore, Lemma 1 implies $\hat{F}_{M N}\left(t \mu_{2}(M N)\right)=\hat{F}_{M}\left(t \mu_{2}(M)\right) \hat{F}_{N}\left(t \mu_{2}(N)\right)$, and Lemma 3 follows.

LEMma 4. Let $p$ and $q$ be primes, and $\alpha$ a positive integer. Then $F_{p^{\alpha}}=F_{q^{\alpha}}$ is an arithmetic distribution with discontinuities at the points

$$
\left\{\left(2 \alpha^{-1} k-1\right)\left((\alpha+2)^{-1} 3 \alpha\right)^{1 / 2}\right\}_{k=0}^{\alpha} .
$$

As $\alpha \rightarrow \infty, F_{p^{\alpha}}$ converges to the uniform distribution $U$ having density $(12)^{-1 / 2} \chi_{[-\sqrt{3}, \sqrt{3}}$.

Lemma 4 follows immediately from Lemma 1 and Theorem 1.

LEMMA 5. Let $F_{j}$ be a sequence of arithmetic distributions with $d_{j}>1$ discontinuities such that $d F_{j}$ is supported in $[-1,1]$. Let $s_{j}$ be the distance between discontinuities of $F_{j}$, and assume $v_{j}$ is a sequence of positive numbers such that

(1) $\sum_{j>J} v_{j}<\frac{1}{4} s_{J} v_{J}$ for $J=1,2, \ldots$,

(2) $\left(\prod_{j=1}^{J} d_{j}\right) \sum_{j>J} v_{j} \rightarrow 0$ as $J \rightarrow \infty$.

Then the convolution $H_{k}(x)=F\left(x / v_{1}\right) * \cdots * F_{k}\left(x / v_{k}\right)$ converges to a singular distribution as $k \rightarrow \infty$.

The proof is easy, and will be omitted.

We now prove Theorem 3. Let $N_{j}$ have prime factorization

$$
N_{j}=p_{j}(1)^{\alpha_{j}(1)} \cdots p_{j}\left(k_{j}\right)^{\alpha_{j}\left(k_{j}\right)}
$$


We abbreviate $p_{j}(i)^{\alpha_{j}(i)}$ as $(j, i)$, and use $\langle x\rangle$ to mean $2^{x}$.

First consider the case $\liminf _{j \rightarrow \infty} \omega\left(N_{j}\right)=K$. By passing to a subsequence and reindexing, we may assume $\omega\left(N_{j}\right)=k$, and $(j, k)>(j, l)$ for $k<l$. Let $v_{j}(k)=$ $\mu_{2}((j, k))\left(\mu_{2}\left(N_{j}\right)\right)^{-1}$, and $M_{j}(k)=(j, k)^{-1} N_{j}$. Assume that $F$ is not a finite point mass distribution.

Repeated use of Lemma 3 gives

$$
\hat{F}_{N_{j}}(t)=\prod_{k=1}^{K} \hat{F}_{(j, k)}\left(v_{j}(k) t\right) .
$$

Since each $v_{j}(k) \in[0,1]$, we may pass to a subsequence and assume $v_{j}(k) \rightarrow v_{k}$ as $j \rightarrow \infty$. If any $v_{k}=0$, then $\hat{F}_{(j, k)}\left(v_{j}(k) t\right) \rightarrow 1$ for all $t$. Hence such a factor can be ignored when considering $\lim _{j \rightarrow \infty} \hat{F}_{N_{j}}$, and so we may assume $v_{k}>0$. We may also pass to a subsequence and assume each $F_{(j, k)}$ in (3.1) converges. Therefore, Theorem 1 implies that either $\alpha_{j}(k) \rightarrow \infty$ or the sequence $\alpha_{j}(k)$ becomes constant, say $\alpha_{j}(k)=\alpha_{k}$, for large $j$. If for all $k, \alpha_{j}(k) \rightarrow \alpha_{k}$, then (3.1) and Lemma 4 give

$$
\hat{F}_{N_{j}}(t) \rightarrow \hat{F}_{\left\langle\alpha_{1}\right\rangle}\left(v_{1} t\right) \cdots \hat{F}_{\left\langle\alpha_{k}\right\rangle}\left(v_{K} t\right),
$$

so that $F$ would be a finite point mass distribution, contrary to hypothesis. Therefore, let $k$ be such that $\alpha_{j}(k) \rightarrow \infty$, and let $M_{j}=M_{j}(k)$. By Lemma 3 we have

$$
\hat{F}_{N_{j}}(t)=\hat{F}_{M_{j}}\left(t \sin \phi_{j}\right) \hat{F}_{(j, k)}\left(t \cos \phi_{j}\right),
$$

where $\cos \phi_{j}=v_{j}(k)$. As $j \rightarrow \infty$, we have $\cos \phi_{j} \rightarrow \cos \phi=v_{k}>0$, and by Lemma $4, \hat{F}_{(j, k)}(t) \rightarrow \hat{U}(t)$. Passing to a subsequence, we have also $\hat{F}_{M_{j}} \rightarrow \hat{H}$ as $j \rightarrow \infty$. Therefore, $F(x)=U(x \sec \phi) * H(x \csc \phi)$.

Note that $\omega\left(M_{j}\right)<k=\omega\left(N_{j}\right)$; so, by redefining $N_{j}$ as $M_{j}$, the above argument can be repeated at most $k-1$ times.

Now consider the case $\omega\left(N_{j}\right) \rightarrow \infty$. Assume that $F$ has no uniform or normal convolution factors, and is not a finite point mass distribution. We will show that $F$ either has a singular convolution factor, or is the limit of a sequence $F_{L_{j}}$ with $\omega\left(L_{j}\right)=O(1)$.

Since $F$ is not normal, Theorem 2 gives the existence of a $\delta>0$ such that, for infinitely many $j, \mu_{\infty}\left(N_{j}\right)\left(\mu_{2}\left(N_{j}\right)\right)^{-1}>\delta$. Passing to a subsequence we may assume this for all $j$. Lemma 3 gives

$$
\hat{F}_{N_{j}}(t)=\hat{F}_{M_{j}(1)}\left(t \sin \phi_{j}\right) \hat{F}_{(j, 1)}\left(t \cos \phi_{j}\right),
$$

where $\cos \phi_{j}=v_{j}(1)$. Inequality (2.3) implies that $v_{j}(1)>\delta / 4$, so we may pass to a subsequence and assume $\cos \phi_{j} \rightarrow \cos \phi=v_{1} \geqslant \delta / 4$ as $j \rightarrow \infty$. If $\sin \phi=0$, then $\hat{F}_{M_{j}(1)}\left(t \sin \phi_{j}\right) \rightarrow 1$ for all $t$. Hence, this factor could be ignored when considering $\lim _{j \rightarrow \infty} F_{N_{j}}$, and $F$ would be the limit of a sequence $F_{L_{j}}$ with $\omega\left(L_{j}\right)=O(1)$ (take $L_{j}=(j, 1)$ ). By passing to a subsequence, we may assume that each factor in (3.2) converges. Since $F$ has no uniform convolution factor, this implies that the sequence $\alpha_{j}(1)$ becomes constant, say $\alpha_{j}(1)=\alpha_{1}$, for large $j$.

If we assume that $F$ is not the limit of a sequence $F_{L_{j}}$ with $\omega\left(L_{j}\right)=O(1)$, then by redefining $N_{j}$ as $M_{j}(1)$, the above argument can be repeated indefinitely. The $k$ th application of the argument produces a subsequence $\hat{F}_{N_{k 1}}, \hat{F}_{N_{k 2}}, \ldots$ of the sequence 
generated at the $k-1$ st stage along which

$$
\hat{F}_{(j, k)}\left(v_{j}(k) t\right) \rightarrow \hat{F}_{\left\langle\alpha_{k}\right\rangle}\left(v_{k} t\right) \text {. }
$$

Let $N_{j}=N_{j j}$ be the diagonal sequence. It follows that

$$
\hat{F}_{N_{j}}(t)=\prod_{k=1}^{k_{j}} \hat{F}_{\left\langle\alpha_{j}(k)\right\rangle}\left(v_{j}(k) t\right),
$$

where for any $k, v_{j}(k) \rightarrow v_{k}$ and $\alpha_{j}(k) \rightarrow \alpha_{k}$ as $j \rightarrow \infty$.

Fatou's Lemma gives

$$
\sum_{k=1}^{\infty} v_{k}^{2} \leqslant \liminf _{j \rightarrow \infty} \sum_{k=1}^{k_{j}} v_{j}^{2}(k)=1 .
$$

Hence there exists a subset $\left\{v_{k^{*}}\right\}_{k=1}^{\infty}$ of the set $\left\{v_{k}\right\}_{k=1}^{\infty}$ satisfying the conditions of Lemma 5 with respect to the distributions $F_{\left\langle\alpha_{k} \cdot\right\rangle}(x)$. Let $f: Z^{+} \rightarrow Z^{+}$be a nondecreasing function satisfying the following conditions:

(A) $f(j) \leqslant k_{j}$ and $\lim _{j \rightarrow \infty} f(j)=\infty$,

(B) $k<f(j)$ implies $\alpha_{j}\left(k^{*}\right)=\alpha_{k^{*}}$ and $\left|v_{j}\left(k^{*}\right)-v_{k^{*}}\right|<2^{-f(j)} v_{k^{*}}$.

Let $M_{j}=\Pi_{k<f(j)}\left(j, k^{*}\right)$, and $\cos \phi_{j}=\left(\mu_{2}\left(N_{j}\right)\right)^{-1} \mu_{2}\left(M_{j}\right)$. Applying Lemma 3, we reorganize (3.3) as

$$
\hat{F}_{N_{j}}(t)=\hat{F}_{M_{j}^{-1} N_{j}}\left(t \sin \phi_{j}\right) \prod_{k<f(j)} F_{\left\langle\alpha_{k} \cdot\right\rangle}\left(v_{j}\left(k^{*}\right) t\right) .
$$

By passing to a subsequence, the first factor on the right-hand side of (3.4) converges to $\hat{H}(t \sin \phi)$ for some distribution $H$. The proof is completed by noting that the second factor converges to

$$
\prod_{k=1}^{\infty} F_{\left\langle\alpha_{k} \cdot\right\rangle}\left(v_{k^{*}} t\right),
$$

which by Lemma 5 is the transform of a singular distribution.

\section{REFERENCES}

1. J. Conway, Functions of one complex variable, Springer-Verlag, New York, 1978.

2. P. Erdös and J. L. Nicolas, Méthodes probabilistes et combinatoires en théorie des nombres, Bull. Sci. Math. France (2) 100 (1976), 301-320.

3. W. Feller, An introduction to probability theory and its applications, Vol. II, Wiley, New York, 1966.

4. D. S. Mitrinovic, Analytic inequalities, Springer-Verlag, New York, 1970.

Department of Mathematics, Texas A \& M University, College Station, Texas 77843

Current address: 2005B Cheshire Drive, Austin, Texas 78723 